\title{
Host Modification of Chlamydiae: Differential Infectivity for Cell Monolayers of Chlamydiae Grown in Eggs and Monolayers
}

\author{
By I. ALLAN AND J. H. PEARCE \\ Department of Microbiology, University of Birmingham, B15 2TT
}

(Received 15 September 1978)

Cell monolayer-grown chlamydiae (CGO) differed from egg-grown organisms (EGO) in their increased spontaneous infectivity relative to centrifuge-assisted infectivity for monolayers. For each population spontaneous: centrifuge-assisted infectivity ratios were constant over a wide dose range. Spontaneous infection increased linearly with time and could not be exhausted from either population by prolonged adsorption; there was no change in infectivity ratios in residual supernatants. Further, one passage of EGO through monolayers gave CGO with stable infectivity properties not increased by further cell passage yet reverting on a single passage in eggs. Spontaneous infection of monolayers with EGO gave progeny with the same infectivity ratios as monolayers infected with EGO by centrifugation. The change in properties following EGO infection of monolayers occurred prior to natural release from cells. We conclude that EGO and CGO are two phenotypically distinct, homogeneous populations. The two infection modes are not properties of subpopulations within EGO and CGO. The relationship of these observations on chlamydiae to other possible host-imposed phenomena is considered.

\section{INTRODUCTION}

Early reports described variability in the adaptation of Chlamydia trachomatis isolated in the chick embryo to growth in monolayers of continuous cell cultures (Collier, 1962; Mitsui et al., 1964). In later work adaptation was achieved more readily (Jenkin, 1966; Mitsui et al., 1967), especially with the adoption of centrifugation to assist infection of monolayers (Gordon et al., 1969). There was no evident change in antigenic properties during adaptation (Gordon et al., 1971).

We have previously developed a quantitative assay for infectivity of the guinea-pig inclusion conjunctivitis strain (GP-IC) of $C$. psittaci using centrifugation to enhance infection of irradiated McCoy cell monolayers (Griffiths et al., 1976). We found that organisms passaged through monolayers or the guinea-pig conjunctiva showed a greater capacity to infect static monolayers (spontaneous infection) than organisms grown in the chick embryo. Here, we present evidence that the increased spontaneous infection of monolayers shown by monolayer-grown GP-IC depends on phenotypic properties conferred by the host chosen for propagation of the organisms.

\section{METHODS}

Organisms grown in eggs (EGO). The agent of guinea-pig inclusion conjunctivitis (GP-IC) was grown in eggs and harvested as previously described (Moore et al., 1974). For purification of organisms from infected yolk sac membranes, we used either Renografin-76 (meglumine diatrizoate and sodium diatrizoate; E. R. Squibb \& Sons, New York, U.S.A.), following the batch procedure of Howard et al. (1974), or KCl (Taverne et al., 1964). Purified organisms were stored at $-70^{\circ} \mathrm{C}$.

Organisms grown in irradiated McCoy cells $(C G O)$. McCoy cell monolayers, $4 \mathrm{~d}$ after irradiation (Allan \& Pearce, 1977), were inoculated with suspensions of GP-IC (EGO) containing approximately 10 inclusion- 


\section{Table 1. Spontaneous and centrifuge-assisted infectivities of egg-and monolayer-grown organisms}

GP-IC samples grown in yolk sacs (EGO) or monolayers (CGO) were inoculated on to monolayers (four replicates per sample) with or without centrifugation. EGO were purified with $\mathrm{KCl}$ or Renografin-76(Ren).

\begin{tabular}{|c|c|c|c|c|}
\hline \multirow[b]{2}{*}{ Expt } & \multirow[b]{2}{*}{ Organisms } & \multicolumn{2}{|c|}{$10^{-4} \times$ Infectivity titre (i.f.u. $\mathrm{ml}^{-1}$ ) } & \multirow[b]{2}{*}{ S:CA } \\
\hline & & $\begin{array}{l}\text { Spontaneous } \\
\text { (S) }\end{array}$ & $\begin{array}{l}\text { Centrifuge- } \\
\text { assisted (CA) }\end{array}$ & \\
\hline 1 & $\begin{array}{l}\text { EGO (KCl) } \\
\text { EGO (Ren) } \\
\text { CGO }\end{array}$ & $\begin{array}{l}2 \cdot 1 \\
0 \cdot 56 \\
3 \cdot 2\end{array}$ & $\begin{array}{r}1600 \\
390 \\
65\end{array}$ & $\begin{array}{l}1: 762 \\
1: 696 \\
1: 20\end{array}$ \\
\hline 2 & $\begin{array}{l}\text { EGO (KCl) } \\
\text { EGO (Ren) } \\
\text { CGO }\end{array}$ & $\begin{array}{l}0.49 \\
0.51 \\
2.9\end{array}$ & $\begin{array}{r}370 \\
400 \\
71\end{array}$ & $\begin{array}{l}1: 755 \\
1: 784 \\
1: 25\end{array}$ \\
\hline 3 & $\begin{array}{l}\mathrm{EGO}(\mathrm{KCl}) \\
\mathrm{CGO}\end{array}$ & $\begin{array}{l}0 \cdot 62 \\
3 \cdot 0\end{array}$ & $\begin{array}{r}410 \\
94\end{array}$ & $\begin{array}{l}1: 661 \\
1: 31\end{array}$ \\
\hline
\end{tabular}

forming units (i.f.u.) per cell. Infection was initiated by centrifugation (Griffiths et al., 1976) of organisms with cell monolayers $\left(1580 \mathrm{~g}, 60 \mathrm{~min}, 35^{\circ} \mathrm{C}\right)$. After $42 \mathrm{~h}$ incubation at $35^{\circ} \mathrm{C}$, supernatant fluid containing naturally released organisms was removed and centrifuged $\left(12000 \mathrm{~g}, 30 \mathrm{~min}, 4{ }^{\circ} \mathrm{C}\right)$. The pellet was resuspended in phosphate-buffered saline containing $0.25 \mathrm{M}$-sucrose (PBS sucrose), centrifugation was repeated and the resulting pellet was resuspended in PBS sucrose $(0 \cdot 1$ to 0.01 of the original volume). CGO were freshly prepared immediately before each experiment in which they were used. Particle numbers were determined by the method of Reeve \& Taverne (1962).

In one group of experiments the spontaneous infectivities of naturally and ultrasonically released CGO were compared. Organisms were prepared by sonication $\left(30 \mathrm{~s}, 0^{\circ} \mathrm{C}, 75 \mathrm{~W}, 3 \mathrm{~mm}\right.$ probe; Rapidis 180 , Ultrasonics Ltd, Shipley, Yorks.); before sonication, naturally released organisms were removed by rinsing monolayers with growth medium $(2 \times 1 \mathrm{ml})$. Subsequent steps in preparation were as for naturally released organisms.

Preparation and culture of irradiated McCoy cell monolayers. These were as previously described (Allan \& Pearce, 1977).

Titration of centrifuge-assisted and spontaneous infectivity of GP-IC in cell monolayers. This was carried out as described by Griffiths et al. (1976), but with a lower centrifugal force of $1580 \mathrm{~g}$. Ratios of organisms to cells were adjusted so that less than $10 \%$ of cells were infected for all titrations reported (to avoid underestimation of infectivity caused by multiple infection of cells). Organism dilutions were prepared in Hanks' balanced salts solution supplemented with $10 \%(\mathrm{v} / \mathrm{v})$ foetal bovine serum (HFBS). In all experiments, samples were titrated on three or four replicate monolayers; in no case did the standard error exceed $10 \%$.

\section{RESULTS}

\section{Differential behaviour of egg-and monolayer-grown organisms in spontaneous infection}

As previously reported (Griffiths et al., 1976), GP-IC inocula grown in the yolk sac of the chick embryo (EGO) showed greatly increased spontaneous infection of cell monolayers after a single passage through cell monolayers (CGO; Table 1). The altered ratio of spontaneous:centrifuge-assisted infectivity reflected a true rise in capacity for spontaneous infection since the inherent infectivities of EGO and CGO populations, judged by centrifugeassisted infectivity per particle, were comparable (particle:centrifuge-assisted infectivity ratios of 12:1 and 6:1 for EGO and CGO preparations, respectively). The previous work had shown that the infectivity ratios for EGO preparations were unaffected by the presence of yolk sac material. This was confirmed and extended by the demonstration that the ratio was unchanged for Renografin-purified preparations (Table 1). Renografin purification gave a 600-fold reduction in the phosphorus content of a lipid extract of purified organisms, compared with that of unpurified organisms. After Renografin purification there was also a marked reduction in the pellet size of preparations which had previously been purified with $\mathrm{KCl}$, with good retention of particle and infectivity content. 


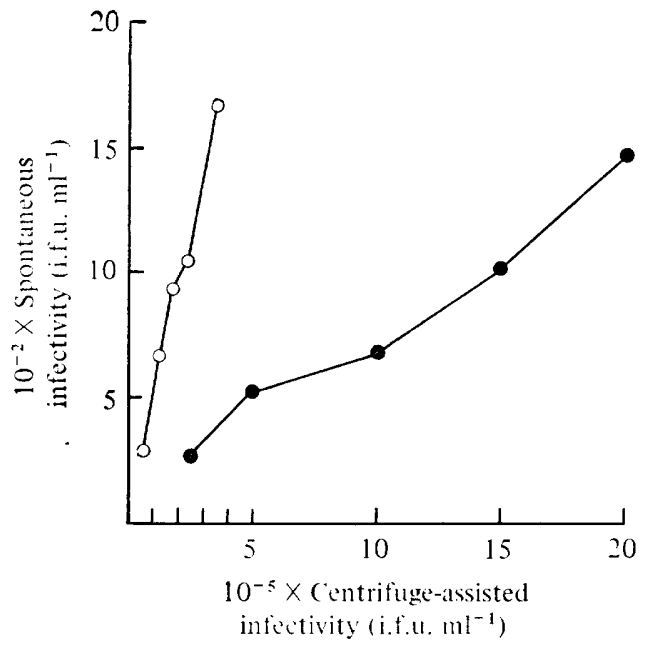

Fig. 1. Relationship between spontaneous and centrifuge-assisted infectivities of EGO (O) and CGO $(O)$ suspensions over a 10-fold dose range. Suspensions were titrated directly for spontaneous infectivity $\left(60 \mathrm{~min}, 35^{\circ} \mathrm{C}, 1 \mathrm{~g}\right)$ or diluted (HFBS) for determination of centrifuge-assisted infectivity $\left(60 \mathrm{~min}, 35^{\circ} \mathrm{C}, 1580 \mathrm{~g}\right)$.

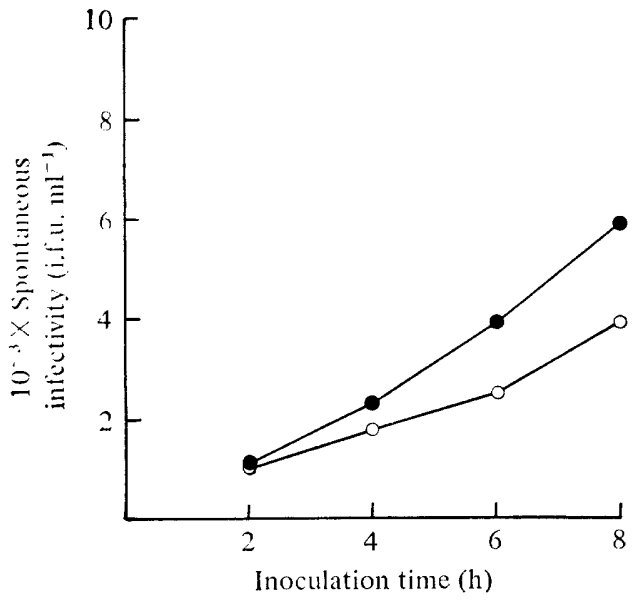

Fig. 2

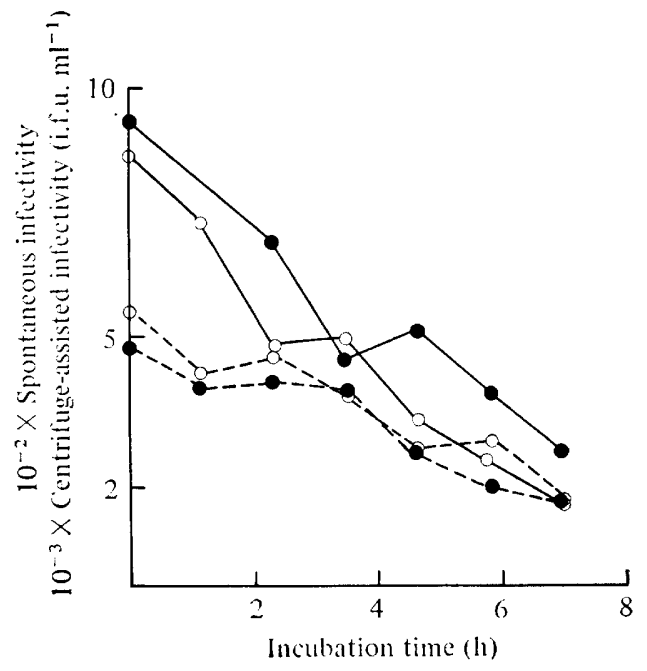

Fig. 3

Fig. 2. Increase in spontaneous infection of cells by EGO (O) and CGO $(O)$ with increasing inoculation time. Suspensions of EGO and CGO were inoculated on to monolayers and incubated at $35^{\circ} \mathrm{C}$. At $2 \mathrm{~h}$ intervals the inoculum was removed from sample monolayers, replaced with growth medium, and the monolayers were incubated at $35^{\circ} \mathrm{C}$ to allow inclusion formation. The total incubation time for monolayers, including the period in the presence of the inoculum, was $28 \mathrm{~h}$. Values have been corrected for thermal inactivation of spontaneous infectivity (see Fig. 3).

Fig. 3. Thermal inactivation of spontaneous infectivity $(-)$ and centrifuge-assisted infectivity $(-)$ ) EGO (O) and CGO (O). Suspensions of EGO and CGO (of the same concentration as in Fig. 2) were incubated at $35^{\circ} \mathrm{C}$. At $70 \mathrm{~min}$ intervals samples were taken and titrated for spontaneous and centrifuge-assisted infectivity on monolayers (three replicates per sample). Suspensions of EGO and CGO were diluted $1: 100$ and $1: 10$, respectively, before titration of centrifuge-assisted infectivity. 
Table 2. Spontaneous infection of monolayers with EGO or CGO and infectivity ratios of supernatants from successive infections

Suspensions of EGO or CGO were incubated with static monolayers for $60 \mathrm{~min}$ at $35^{\circ} \mathrm{C}$. Supernatants were then removed and pooled, samples were taken for measurement of spontaneous and centrifuge-assisted infectivity, and the remainder was used to infect further static monolayers. The process was repeated once more for CGO and twice more for EGO.

$\begin{array}{lcccc}\text { Organisms } & \begin{array}{c}\text { Supernatant } \\ \text { (successive) }\end{array} & \begin{array}{c}\text { Spontaneous } \\ (\mathbf{S})\end{array} & \begin{array}{c}\text { Centrifuge- } \\ \text { assisted (CA) }\end{array} & \text { S:CA } \\ \text { EGO } & 1 & 2 \cdot 9 & 2000 & 1: 690 \\ & 2 & 2 \cdot 2 & 1900 & 1: 864 \\ & 3 & 0 \cdot 87 & 790 & 1: 906 \\ \text { CGO } & 4 & 0 \cdot 75 & 710 & 1: 946 \\ & 1 & 3 \cdot 7 & 100 & 1: 27 \\ & 2 & 2 \cdot 2 & 89 & 1: 40 \\ & 3 & 1 \cdot 6 & 59 & 1: 37\end{array}$

\section{Relationship between spontaneous and centrifuge-assisted infection}

In general, it appears that, for most chlamydiae, spontaneous and centrifuge-assisted infection are properties of one and the same organism. Nevertheless, before exploring the differences between EGO and CGO, it seemed desirable to show that the two infection modes did not reflect distinct subpopulations within each preparation. This was confirmed by the finding that infectivity ratios were constant over a 10 -fold dose range, for both EGO and CGO (Fig. 1). The amount of spontaneous infection increased linearly with incubation time (Fig. 2) when correction was made for thermal inactivation of infectivity during incubation (Fig. 3). Evidently, spontaneously infecting organisms were not exhausted from the inoculum population by prolonged adsorption. It followed that considerable numbers of organisms exhibiting both spontaneous and centrifuge-assisted infectivity should be present in supernatants. This was tested by measuring the infectivity ratios of residual organisms, after spontaneous infection, by inoculation of fresh monolayers with successive supernatants. Four successive supernatants from an EGO inoculum and three from a CGO inoculum were titrated (Table 2). Infectivity ratios showed a small change which was attributed to the somewhat greater thermal inactivation rate for spontaneous than for centrifuge-assisted infectivity (see Fig. 3).

Essentially, the incubation of organisms with static monolayers, which allowed spontaneous infection, did not lead to selective loss of spontaneously infecting organisms from inocula. Also, progeny organisms derived from spontaneous infection of monolayers by CGO were shown to have similar spontaneous: centrifuge-assisted infectivity ratios $(1: 27$ and $1: 38$ in two experiments) to the starting inoculum. Thus, spontaneous infection did not lead to progeny capable only of spontaneous infection.

\section{Phenotypic conversion by growth of organisms in eggs or monolayers}

These findings indicated that spontaneous and centrifuge-assisted infection were both properties of the same organisms. It remained to determine whether the increased spontaneous infectivity displayed by organisms grown in monolayers was a result of population selection or an acquired phenotypic property.

EGO were passaged through monolayers and the infectivity properties of samples of progeny were examined after two further passages in cells or re-passage in eggs. Passage through monolayers resulted in an immediate increase in the capacity of organisms for spontaneous infection of monolayers which was stable in further monolayer passage but was reversed by a single passage in eggs (Table 3). Further experiments showed that the 


\title{
Table 3. Change in infectivity ratios during passage of EGO in monolayers and re-passage in eggs
}

\begin{abstract}
EGO were passaged three times in monolayers with centrifugation; progeny samples from each passage were titrated for spontaneous and centrifuge-assisted infectivity. In a separate experiment, CGO (produced after a single passage of EGO in monolayers) were passaged once in eggs and the progeny were titrated for spontaneous and centrifuge-assisted infectivity.
\end{abstract}

\section{Organisms}

EGO

Monolayer passage 1

Monolayer passage 2

Monolayer passage 3

CGO

Egg passage 1

$\overbrace{\text { Spontaneous }}^{10^{-4} \times \text { Infectivity titre (i.f.u. } \mathrm{ml}^{-1} \text { ) }}$

(S)

$5 \cdot 7$

$5 \cdot 7$

6.4

$1 \cdot 3$

$2 \cdot 2$

$$
\text { assisted (CA) }
$$

$\overline{210}$

140

55

1900
S:CA

$1: 784^{*}$

$1: 37$

$1: 22$

$1: 42$

$1: 27^{*}$

$1: 864$

* Infectivity ratios determined in earlier experiments.

increased spontaneous infectivity was acquired at some stage prior to release of organisms from cells. After inoculation of monolayers with EGO, progeny released naturally or by ultrasonic treatment of monolayers showed similar spontaneous:centrifuge-assisted infectivity ratios (1:20 and $1: 23$, respectively). Ultrasonic treatment of organisms naturally released from cells did not affect their spontaneous:centrifuge-assisted infectivity ratio $(1: 25)$.

\section{DISCUSSION}

The evidence indicates that EGO and CGO are two homogeneous populations in respect of their infectivity properties, differing phenotypically in their capacity for spontaneous infection. Calculation from the data compiled for Table 2 shows that after $1 \mathrm{~h}$ incubation with static monolayers more than $96 \%$ of inoculum infectivity (assessed by centrifugation) remained in supernatants. Thus, low spontaneous infectivity reflects a low affinity of organisms for the cell surface. However, the greater spontaneous infectivity shown by CGO cannot strictly be interpreted as arising from higher affinity. For both preparations, more organisms may become cell-associated than give rise to infection. An accurate assessment would require use of radiolabelled organisms to measure associated organisms; the small difference in unassociated infectivity in supernatants could not be measured with sufficient precision.

The ratios of spontaneous:centrifuge-assisted infectivity found here for EGO and CGO are lower than those previously reported (1:3500 and 1:124, respectively; Griffiths et al., 1976). The variation arises from alteration in the levels of spontaneous infectivity and, while not affecting relative properties of EGO and CGO, appears to depend on properties of the cell system, which change with the serum batch used for cell cultivation. The effect probably results from subtle alteration in cell surface properties which are modulated by the serum (Allan \& Pearce, 1977).

Thermal inactivation was the same for EGO and CGO but more rapid for spontaneous than centrifuge-assisted infection (Fig. 3 ). This suggests possible differences in the organism cell interaction for the two infection modes - assuming that events are comparable subsequent to ingestion. This is in accord with our observation that infection enhancement by centrifugation depends on the induction of cell surface changes (Allan \& Pearce, 1979a).

Renografin-purified EGO showed the same low spontaneous infectivity as crude yolk sac preparations. This strongly suggests that the low infectivity was not the result of inhibitory yolk sac material present in EGO. It does not, however, exclude the possibility 
that some yolk sac component becomes firmly bound to the chlamydial surface; we present evidence in the following paper that EGO have an associated egg antigen (Allan \& Pearce, $1979 b$ ).

The altered property of CGO spontaneous infectivity is acquired before the organisms are released from cells. How release of GP-IC occurs is not known. In other C. psittaci strains it occurs by both cell lysis and 'budding' mechanisms (Doughri et al., 1972); the latter might lead to surface changes in released organisms.

Alteration in the capacity for spontaneous infection imposed by the host could have significance for chlamydial infection in vivo. Kordova et al. (1972) found differences between monolayer- and egg-grown C. psittaci, strain 6BC; monolayer-grown organisms multiplied in cell monolayers with widespread cytotoxicity whereas egg-grown organisms infected monolayers without damage. However, the relationship between the two forms of agent was not clarified; frequent cell passage may have resulted in substantial selection. In earlier studies with a trachoma strain of $C$. trachomatis (Moore et al., 1970) we observed that the numbers of infected epithelial cells increased during passage of egg-grown organisms through the baboon conjunctiva. Such effects might be explained by the change in the organism that we describe here. We have not, however, observed significant differences in initiation of infection in the guinea-pig eye with EGO, CGO or GP-IC grown in the guinea-pig conjunctiva (S. Ainsworth \& J. H. Pearce, unpublished). Kuo et al. (1975) reported that $C$. trachomatis isolated from the conjunctiva of a neonate and the cervix of the mother (presumed origin of the neonatal infection) differed in their ability to infect HeLa cell monolayers. They suggested that the difference may have been due to alteration of the organisms by growth in different tissues. Kuo et al. (1975) also reported that antigenically identical organisms isolated simultaneously from different tissues (conjunctiva and genital tract) differed in their ability to infect HeLa cells. Whether these differences depend on changes similar to those described here awaits further investigation.

\section{A. was in receipt of an MRC research studentship.}

\section{REFERENCES}

Allan, I. \& Pearce, J. H. (1977). Serum modulation of cell susceptibility to chlamydial infection. FEMS Microbiology Letters 1, 211-214.

Allan, I. \& Pearce, J. H. (1979a). Modulation by centrifugation of cell susceptibility to chlamydial infection. Journal of General Microbiology 111, 87-92.

Allan, I. \& Pearce, J. H. (1979b). Host modification of chlamydiae: presence of an egg antigen on the surface of chlamydiae grown in the chick embryo. Journal of General Microbiology 112, 61-66.

Collier, L. H. (1962). Growth characteristics of inclusion blennorrhea virus in cell cultures. Annals of the New York Academy of Sciences 98, 42-49.

Doughri, A. M., Storz, J. \& Altera, K. P. (1972). Mode of entry and release of chlamydiae in infections of intestinal epithelial cells. Journal of Infectious Diseases 126, 652-657.

Gordon, F. B., HARPER, I. A., QuAN, A. L., Treharne, J. D., DWYer, R. St. C. \& Garland, J. A. (1969). Detection of chlamydia (Bedsonia) in certain infections of man. I. Laboratory procedures: comparison of yolk sac and cell culture for detection and isolation. Journal of Infectious Diseases 120, 451-462.
Gordon, F. B., Nichols, R. L. \& QuaN, A. L. (1971). Immunotyping of Chlamydia trachomatis with fluorescent antibody: retention of immunospecificity in cell culture passage, and typing with infected cell monolayers. In Trachoma and Related Disorders, pp. 358-362. Edited by R. L. Nichols. Amsterdam: Excerpta Medica.

Griffiths, M. S., Ainsworth, S. A. \& Pearce, J. H. (1976). Infectivity titration of guinea-pig inclusion conjunctivitis agent in irradiated McCoy cells. Journal of General Microbiology 95, 249-256.

Howard, L., Orenstein, N. S. \& King, N. W. (1974). Purification on Renografin density gradients of Chlamydia trachomatis grown in the yolk sac of eggs. Applied Microbiology 27, 102-106.

Jenkin, H. M. (1966). The continuous passage of agents of trachoma in cell culture. 1. Characteristics of TW-3 and Bour strains of trachoma cultivated in serial passage in HeLa cells. Journal of Infectious Diseases 116, 390-399.

Kordova, N., Poffenroth, L. \& Wilt, J. C. (1972). Lysosomes and the 'toxicity' of Rickettsiales. III. Response of $\mathrm{L}$ cells infected with egg-attenuated C. psittaci strain 6BC. Canadian Journal of Microbiology 18, 1343-1348.

Kuo, C. C., Wang, S. P. \& Grayston, J. T. (1975). 
Comparative infectivity of trachoma organisms in HeLa 229 cells and egg cultures. Infection and Immunity 12, 1078-1082.

Mitsui, Y., Fujimoto, M. \& Kajima, M. (1964). Development and morphology of trachoma agent in the yolk sac cell as revealed by electron microscopy. Virology 23, 30-45.

Mitsui, Y., Kitamuro, T. \& Fujimoto, M. (1967). Adaption of TRIC agents to tissue culture and characteristics of tissue culture adapted variants. American Journal of Ophthalmology 63, 1191-1205. Moore, J. E., Griffiths, M. S. \& Pearce, J. H. (1970). Virulence of trachoma-inclusion conjunctivitis agent after passage in baboons. British Journal of Experimental Pathology 51, 298-304.
Moore, J. E., Griffiths, M. S. \& Pearce, J. H. (1974). Chlamydial infection of conjunctival tissues in culture. British Journal of Experimental Pathology 55, 396-405.

Reeve, P. \& Taverne, J. (1962). A simple method for total particle counts of trachoma and inclusion blennorrhoea viruses. Nature, London 195, 923 924.

Taverne, J., Blyth, W. A. \& Reeve, P. (1964). Toxicity of the agents of trachoma and inclusion conjunctivitis. Journal of General Microbiology 37, 271-275. 